\title{
Detection of Microembolic Signals in the Common Carotid Artery Using Doppler Sonography in the Porcine Model of Acute Heart Failure Treated by Veno-Arterial Extracorporeal Membrane Oxygenation
}

\author{
D. JANÁK ${ }^{1,2}$, P. HÁLA ${ }^{1}$, M. MLČEK ${ }^{1}$, M. POPKOVÁ ${ }^{1}$, S. LACKO ${ }^{1}$, J. KUDLIČKA ${ }^{1}$, \\ O. KITTNAR ${ }^{1}$
}

${ }^{1}$ Department of Physiology, First Faculty of Medicine, Charles University, Prague, Czech Republic, ${ }^{2}$ Department of Cardiovascular Surgery, Second Faculty of Medicine, Charles University and University Hospital Motol, Prague, Czech Republic

Received March 9, 2017

Accepted November 8, 2017

\begin{abstract}
Summary
Veno-arterial extracorporeal membrane oxygenation (VA-ECMO) is a method used for the treatment most severe cases of decompensated heart failure. The purpose of this study was to evaluate the risk of the formation of microembolisms during VA-ECMO-based therapy. Heart failure was induced with simultaneous detection of microembolisms and the measurement of blood flow rate in the common carotid artery (CCA) without VA-ECMO $(0 \mathrm{l} / \mathrm{min})$ and at the VA-ECMO blood flow rate of 1, 2, 3 and $4 \mathrm{l} / \mathrm{min}$. If embolisms for VA-ECMO $0 \mathrm{l} / \mathrm{min}$ and the individual regimes for VA-ECMO 1, 2, 3, 4l/min are compared, a higher VA-ECMO flow rate is accompanied by a higher number of microembolisms. The final microembolism value at $16 \mathrm{~min}$ was for the VA-ECMO flow rate of $0 \mathrm{l} / \mathrm{min} 0.0(0,1)$, VA-ECMO I/min 7.5 (4, 19), VA-ECMO $2 \mathrm{l} / \mathrm{min} 12.5(4,26)$, VA-ECMO $3 \mathrm{l} / \mathrm{min}$, $21.0(18,57)$ and VA-ECMO $4 \mathrm{l} / \mathrm{min}, 27.5(21,64)$. Such a comparison is statistically significant if VA-ECMO $0 \mathrm{vs} .4 \mathrm{l} / \mathrm{min}$ $p<0.0001,0$ vs. $3 \mathrm{l} / \mathrm{min} p<0.01$ and 1 vs. $4 \mathrm{l} / \mathrm{min} p<0.01$ are compared. The results confirm that high VA-ECMO flow rates pose a risk with regards to the formation of a significantly higher number of microemboli in the blood circulation and that an increase in blood flow rates in the CCA corresponds to changes in the VA-ECMO flow rates.
\end{abstract}

\section{Key words}

Extracorporeal membrane oxygenation • Microembolic signal • Signal • Porcine model

\begin{abstract}
Corresponding author
D. Janák, Department of Physiology, First Faculty of Medicine, Charles University, Albertov 5, 12800 Prague 2, Czech Republic. E-mail: janakdavid@seznam.cz
\end{abstract}

\section{Introduction}

The application of invasive diagnostic and also therapeutic methods in the cardiovascular system poses a risk with regards to the formation of embolisms and microembolisms, which affects a patient's neurological status. In general clinical practice, the use of transcranial Doppler sonography (TCD) has become well-established practice for the monitoring and evaluation of the blood flow rate through cerebral arteries and for the detection of microembolisms in various sites of the circulation. The core of the TCD methodology and its application to the detection of embolisms in blood vessels is a transient increase in the Doppler signal. In 1965, Austen et al. were the first to suggest that such a transient increase in the Doppler signal corresponded to the presence of microembolisms. They described this event happening during the introduction of extracorporeal circulation in a patient when microbubbles appeared in their arterial line (Austen et al. 1965). This work was followed by the measurement of flow rates in the arteries in the circle of Willis through the cranial bone using TCD technology, as performed by Aaslid (Aaslid et al. 1982). A few years later, the method was introduced as the technological 
means by which to diagnose intracerebral vascular pathology. Microembolisms appear in the Doppler spectrum as a short, high-intensity signal which occurs transiently. This signal is defined as a high-intensity transient signal (HITS). That such signals represent microemboli has been reconfirmed by in vitro methods in animal models through the application of artificial emboli in cerebral vessels (Droste et al. 1994). HITSs have been repetitively identified in humans during various interventional procedures in blood vessels. The problem with HITS detection is to distinguish these signals from random artefacts. The issue was dealt with by a consensus of specialists (Consensus Committee of the Ninth International Cerebral Haemodynamic Symposium, 1995; followed by the Consensus on Microembolus Detection by TCD International Consensus Group on Microembolus Detection, 1998) which define a microembolism as a signal corresponding to a different material having different acoustic impedance in blood vessels using specific criteria imposed on the signal. By definition, the microembolic signal (MES) must have the following characteristics: change in the Doppler spectrum characterized by the time behaviour shorter than $300 \mathrm{~ms}$, minimum amplitude higher than $3 \mathrm{~dB}$ against the Doppler background, typical "chirping" noise and the unpredictability of the signal (Ringelstein et al. 1998). A $2 \mathrm{MHz}$ probe is usually used for the signal recording. The method is based on a Fast Fourier Transform (FFT) and a pulse-Doppler signal display. The middle cerebral artery is generally used for the assessment of emboli in the cerebral blood vessels. According to various publications, microemboli are detected in other arteries, for example, also in peripheral arteries (Kudo et al. 2002). The fundamental finding was that the presence of asymptomatic MES is indicated as an important factor associated with an increased risk of damage to the central nervous system (CNS) and the development of brain ischemia (Markus et al. 2005). The detection of microembolisms in the CNS is used in clinical practice in particular for the monitoring of hazardous procedures during which microembolisms can develop. In practice, it has been discovered that signals from the CNS arteries correspond to microemboli in cases of atrial fibrillation, intracardiac thrombi, carotid artery endarterectomy and acute myocardial infarction (Nadareishvili et al. 1999). Today, in the era of advanced cardiac care, VA-ECMO support is used to treat decompensated heart failure. VA-ECMO represents an effective circulation support in the case of refractory circulatory shock (Hála et al. 2016).
This invasive therapeutic method is high-risk due to the possible development of microembolisms. The goal of our experimental study was to ascertain, quantify and describe the development of embolisms (represented by the MES signal) under various VA-ECMO support regimes. Our work also included the evaluation of a change in flow rate through the CCA under various VA-ECMO support regimes.

\section{Methods}

The protocol was designed to include the simulation of the risk of development of microembolisms and the ultrasonic Doppler detection thereof, and the measurement of blood flow rate in the CCA during heart failure therapy by VA-ECMO introduced through a peripheral access from the femoral blood vessels. All procedures were performed on 8 female porcine animals (Sus scrofa domestica, Large White $\times$ Landrace crossbreed) under intensive care, whose age ranged from 4-5 months, with an median weight of $52 \mathrm{~kg}(\min 49, \max 56)$, in an accredited experimental laboratory by a group of specialists. The experimental protocol was reviewed and approved by the Institutional Animal Expert Committee of the First Faculty of Medicine, Charles University and was performed in accordance with Act No. 246/1992 Coll., on the protection of animals against cruelty. All animals were treated and cared for in accordance with the Guide for the Care and Use of Laboratory Animals published by the US National Institutes of Health (NIH Publication No. 85-23, 1985).

\section{Anesthesia and monitoring}

After intramuscular premedication and sedation (azaperone 1-2 mg/kg i.m., atropine $10 \mu \mathrm{g} / \mathrm{kg}$ i.m., ketamine $20 \mathrm{mg} / \mathrm{kg}$ i.m.), cannulation of the marginal ear artery, and $100 \%$ preoxygenation by facial mask, general anaesthesia was started using propofol $(2 \mathrm{mg} / \mathrm{kg}$ i.v.) followed by orotracheal intubation. Mechanical ventilation was introduced by Intellivent - ASV closedloop system (G5, Hamilton Medical, Switzerland). Sp02 was maintained at 95-97\% and end-tidal $\mathrm{CO}_{2}$ $38-42 \mathrm{~mm} \mathrm{Hg}$ adjusted according to the current metabolic parameters. Total intravenous anesthesia was maintained by the continuous administration of propofol $(6-12 \mathrm{mg} / \mathrm{kg} / \mathrm{h})$ and morphine $(0.1-0.2 \mathrm{mg} / \mathrm{kg} / \mathrm{h})$. The depth of anesthesia was monitored by photoreaction, spontaneous movements and bispectral monitoring index. 
The values of the central venous pressure were maintained by saline solution within the range 4$6 \mathrm{~mm} \mathrm{Hg}$. The jugular vein and the left carotid artery were punctured and intravascular approaches were ensured by standard percutaneous intraluminal sheaths. The distal part of the right carotid was surgically exposed and a circular flow rate probe of appropriate sizes attached (3PSB, 4PSB or 6PSB, Scisense, Transonic Systems, USA) to obtain continuous blood flow measurements. The ultrasound probe for the detection of microembolisms (TCD Digi-Lite ${ }^{\mathrm{TM}}$ Rimed) was attached to the proximal portion of the right CCA using a special external adjustable holder. Anticoagulation was performed by unfractionated heparin, a bolus $(100 \mathrm{IU} / \mathrm{kg}$ i.v.), followed by continuous infusion (40-50 IU/ $\mathrm{kg} / \mathrm{h}$ ) with the target level of activating clotting time of (ACT) 210-290 s (the values were checked at the beginning and at the end of the measured intervals using Hemochron Junior+, Edison, USA). Bedside monitoring (LifeScope TR, Nihon Kohden, Japan) included continuous pressure recording invasively through the femoral artery, central venous pressure (TruWave, Edwards Lifesciences, USA), ECG, capnometry, pulse oximetry. Mixed venous oximetry (SvO2), continuous cardiac output ( $\mathrm{CCO})$ and temperature from the pulmonary artery were measured by Swan-Ganz Combo V catheter (Vigilance monitor, Edwards Lifesciences, USA). Intracardiac and transthoracic echocardiography probes (AcuNav IPX8, Acuson P5-1 and X300 ultrasound system, Siemens, USA) were used for 2D and color Doppler imaging. Gas parameters were evaluated by a bedside analysis system (AVL Compact 3, Roche Diagnostics, Germany).

\section{Heart failure}

Prior to the commencement of the protocol, acute heart failure was developed. A percutaneous transluminal angioplasty (PTA) balloon $4 \times 20 \mathrm{~mm}$, shaft $80 \mathrm{~cm}, 0.035$ " (Powerflex Pro, Cordis) was introduced via the femoral artery into the ramus circumflexus, followed by a PTA balloon $4 \times 20 \mathrm{~mm}$, shaft $80 \mathrm{~cm}$, 0.035" (Powerlex Pro, Cordis) into the ramus intervenricularis anterior with insufflation of both the balloons (10 ATM). A monitored 5-minute ischemia was instigated in the left-side coronary blood vessels. Based on the monitoring of $\mathrm{CCO}$, arterial pressure and $\mathrm{ECG}$, gradual desufflation of the balloons was executed and heart failure ascertained by echocardiography. The hemodynamics of the failing circulation was denoted by arterial hypotension due to poor contractility, whereby
CCO was reduced to approximately $50 \%$ of the healthy animal's expected normal value.

\section{VA-ECMO}

After full heparinization, ACT 210-290 s, ECMO cannulas were unilaterally introduced percutaneously using the Seldinger technique into the left femoral artery and vein along with a $23 \mathrm{~F}$ cannula (Medtronic, USA) via the femoral vein into the right atrium and an $18 \mathrm{~F}$ cannula (Medtronic, USA) into the descending thoracic aorta by the femoral artery approach (Oštádal et al. 2016). The cannulas were connected with the ECMO circulation, which consisted of a blood pump (Levitronix Centrimag, Levitronix, USA) and an oxygenator (Quadrox, Maquet, Germany). At first, the ECMO circuit was filled with $500 \mathrm{ml}$ of normal saline solution with 2,500 IU of unfractionated heparin. Extracorporeal blood flow was registered by a separate circumjacent flow probe (ME 9PXL, Transonic Systems, USA) attached to the ECMO outflow cannula. The analysis of the blood gases was continuously monitored (CDI Blood Parameter Monitoring System 500, Terumo Cardiovascular System Corporation, USA). The partial oxygen pressure and air flow rates were repetitively adjusted to maintain $\mathrm{pO}_{2} 100-120 \mathrm{~mm} \mathrm{Hg}$, $\mathrm{pCO}_{2}$ 35-45 mm Hg and $\mathrm{pH}$ 7.35-7.45 during the entire experiment.

\section{Doppler-ultrasound for detection of microembolisms and} the evaluation of flow rate in the CCA

After surgical preparation of the distal portion of the CCA, a circular flow rate probe of the relevant size (3PSB, 4PSB or 6PSB, Scisense, Transonic Systems, USA) was attached to the distal CCA and continuous blood flow measurements were executed. The assessment was performed off-line. A standard TCD device-TCD Digi-Lite $^{\mathrm{TM}}$ Rimed multigate 2-MHz probe, was used for the detection of microemboli. The probe was attached to the animal's neck using a special external adjustable holder with the target in the proximal right CCA. The device, equipped with automatic detection of microemboli and capable of discriminating artefacts, was employed according to its default settings. The detection of emboli complied with the worldwide consensus on MES signal detection of 1995 and 1998. The angle between the probe and the artery for detection was approximately 60 degrees. The depth of detection was selected between $5-6 \mathrm{~cm}$ to acquire the optimal signal of the flow rate wave of the respective artery, whereby 
a light blue color corresponded to the background suitable for the detection of embolisms. During the measurements we performed an on-line analysis of the number of emboli. The detection of microembolisms and the measurements of blood flow rate in the CCA were performed based on the protocol, basically upon heart failure, without VA-ECMO $(0 \mathrm{l} / \mathrm{min})$ and under the individual VA-ECMO regimes (1, 2, 3, 4 1/min.)

\section{Experimental protocol}

At least $30 \mathrm{~min}$ after the development left-sided heart failure, under full systemic heparinization, ACT 210-290 s, simultaneous repeated continuous measurement (every minute for $16 \mathrm{~min}$ ) of microembolisms was commenced using the $2 \mathrm{MHz}$ TCD probe in the area of the right CCA together with the continuous measurement of the blood flow rate in the right CCA using a circular probe of the respective size without the presence of VA-ECMO (0 1/min), and in addition, at least $30 \mathrm{~min}$ after the introduced VA-ECMO, ACT 210-290 s, under the flow rates of VA-ECMO $1 \mathrm{l} / \mathrm{min}$, VA-ECMO $2 \mathrm{l} / \mathrm{min}$, VA-ECMO $3 \mathrm{l} / \mathrm{min}$ and VA-ECMO 4 l/min.

\section{Statistical analysis}

For data processing the software applications SPSS 13.0 and GraphPad Prism 6 were employed. The measured numbers of emboli did not have a normal distribution, which was ascertained on the basis of the Shapiro-Wilk normality test. Friedman's non-parametric test followed by Dunn's test was used to test the difference in the number of emboli between the five ECMO regimes and compare the number of emboli between all pairs of the ECMO regimes, namely for each of the 16 measurements. Friedman's test followed by Dunn's test was used again to test whether the average blood flow rates in the common carotid artery differed for the individual ECMO regimes. All data were presented as the median (minimum, maximum) of the measured values. The value $p \leq 0.05$ was regarded as significant.

\section{Results}

The measurements were performed on 8 porcine animals during heart failure, without introduced VA-ECMO, whereby the final MES value at $16 \mathrm{~min}$ (cumulative amount of emboli, basal parameters) was $0.0(0,1)$, for the VA-ECMO flow rate of $11 / \mathrm{min}$, $7.5(4,19)$, for the VA-ECMO flow rate of $21 / \mathrm{min}$,
$12.5(4,26)$, for the VA-ECMO flow rate of $31 / \mathrm{min}$, $21.0(18,57)$ and for the VA-ECMO flow rate of $41 / \mathrm{min}$, 27.5 (21, 64) (Fig. 1). Based on the evaluation of all 16 measurements, the statistical numbers of emboli significantly differed for the individual VA-ECMO regimes at the significance level $p<0.0001$. When comparing the number of emboli during the basic measurements -without VA-ECMO (0 1/min) and during the individual VA-ECMO flow rate regimes (1, 2, 3, $4 \mathrm{l} / \mathrm{min}$ ), it is evident that a higher number of microemboli were detected at higher flow rates of the VA-ECMO pump. Such a comparison is statistically significant if the VA-ECMO flow rates 0 vs. $41 / \mathrm{min}$ $\mathrm{p}<0.0001$, 0 vs. $31 / \mathrm{min} \mathrm{p}<0.01$ and 1 vs. $41 / \mathrm{min} \mathrm{p}<0.01$ are compared. For all the remaining tests between the pairs of ECMO regimes, a statistically significant difference at the selected significance level of 0.05 could not be proven (Table 1.1). The median of the average flow rate in the CCA during the given measured time intervals for VA-ECMO $0 \mathrm{l} / \mathrm{min}$ was $205.5 \mathrm{ml} / \mathrm{min}$ (181, 231), for VA-ECMO $11 / \mathrm{min}, 245.5 \mathrm{ml} / \mathrm{min}(229,267)$, for VA-ECMO $21 / \mathrm{min}, 329.0 \mathrm{ml} / \mathrm{min}$ (306, 339), for VA-ECMO $31 / \mathrm{min}, 456.0 \mathrm{ml} / \mathrm{min}(441,475)$, and for VA-ECMO 4 1/min, $512.0 \mathrm{ml} / \mathrm{min}$ (481, 541) (Fig. 1). The median of the average blood flow rates in the CCA during the measured time interval significantly differed from each other for the individual VA-ECMO regimes at the significance level $\mathrm{p} \leq 0.0001$. When comparing the flow rates in the CCA under the various VA-ECMO regimes - the VA-ECMO regimes are 0 vs. $41 / \mathrm{min}$ $\mathrm{p}<0.0001$, 0 vs. $31 / \mathrm{min} \mathrm{p}<0.01$, 0 vs. $21 / \mathrm{min} \mathrm{p}<0.05$, 0 vs. $1 \mathrm{l} / \mathrm{min} \mathrm{p}>0.05,1$ vs. $4 \mathrm{l} / \mathrm{min} \mathrm{p}<0.01,1$ vs. $31 / \mathrm{min}$ $\mathrm{p}>0.05,1$ vs. $2 \mathrm{l} / \mathrm{min} \mathrm{p}>0.05,2$ vs. $3 \mathrm{l} / \mathrm{min} \mathrm{p}>0.05,2$ vs. $3 \mathrm{l} / \mathrm{min} \mathrm{p}>0.05$ and 3 vs. $4 \mathrm{l} / \mathrm{min} \mathrm{p}>0.05$ (Table 1.2 ).

\section{Discussion}

MES-focused Doppler-ultrasound monitoring is a very effective non-invasive method for the monitoring of changes in the area of blood vessels supply. By using this method, we can carry out an on-line assessment of the pathological changes and microembolisms in various vascular circulation locations. VA-ECMO is an invasive lifesaving therapeutic method (Chia-Hsuin et al. 2016) which is high-risk due to the development of microembolisms and their propagation into the CNS. Complications related to ECMO support are frequent and increase the total morbidity and mortality in patients (Makdisi et al. 2015). 

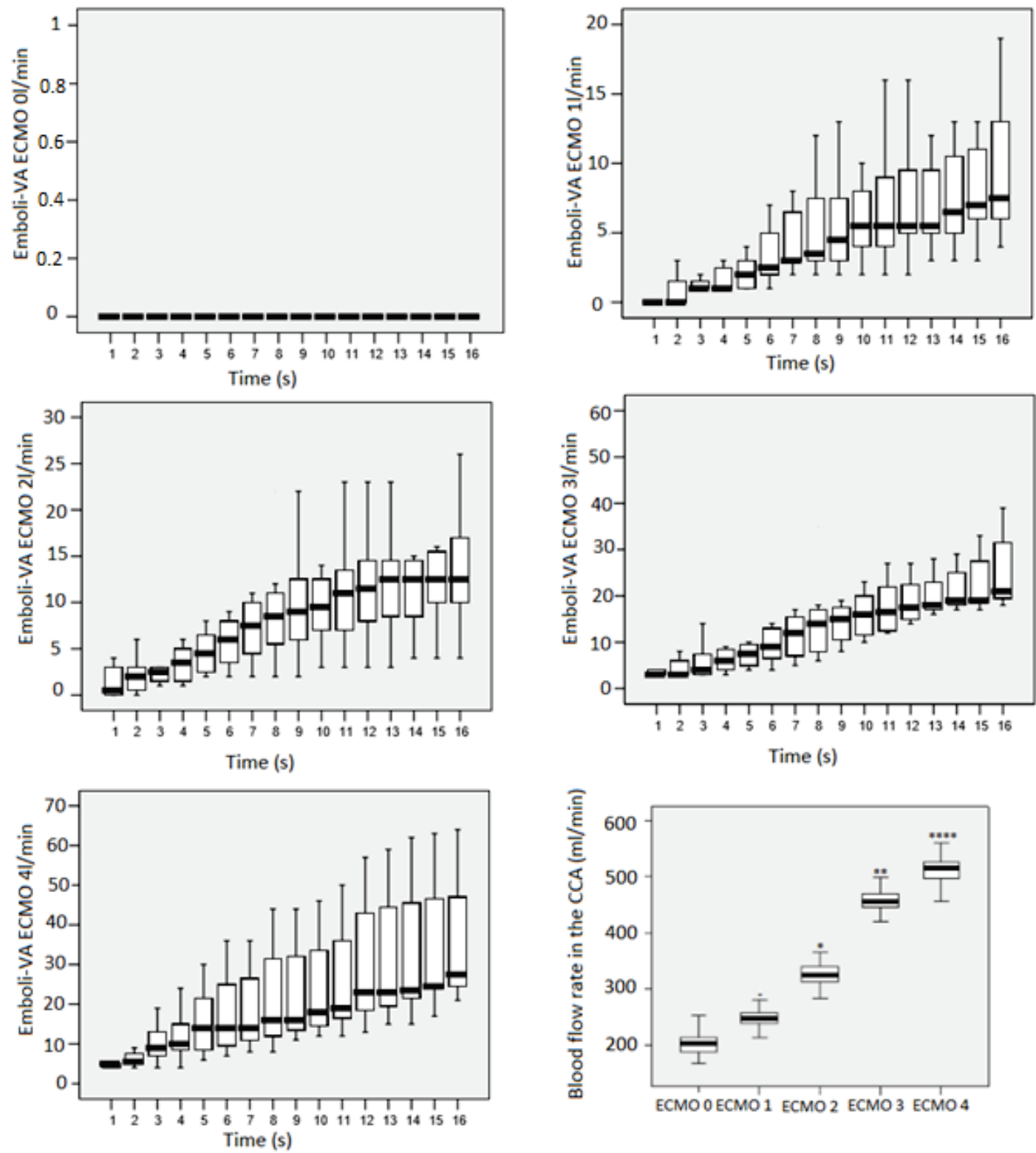

Fig. 1. The number of emboli under VA-ECMO $0,1,2,3$ and $4 \mathrm{l} / \mathrm{min}$ and blood flow rate in the CCA depending on the VA-ECMO flow rate (cumulative amount of emboli/min). - no significant difference, * significant at a significance level of 0.05 , ** significant at a significance level of $0.01, * * *$ significant at a significance level of $0.001, * * * *$ significant at a significance level of 0.0001 , marks significant change from $\mathrm{VA}-\mathrm{ECMO} 0 \mathrm{l} / \mathrm{min}$.

The most frequent complications with ECMO support are hemorrhage, systemic thromboembolism and septic complications (Bartlett et al. 2010). The presence of artificial material in the inlet and outlet cannulas of the ECMO set has a pro-coagulation character. According to references, ECMO support, even at full heparinization, results in many serious thrombotic complications. Thrombotic formations in the tips of cannulas, thrombosis in the aortic root, as well as intracardiac presence of thrombi, have been described (Madershahian et al. 2006). The total risk of complications and the risk of thromboembolisms is higher in VA-ECMO compared to VV-ECMO due to the direct blood supply to the systemic arterial circulation (Mateen et al. 2011). Based on available publications, the risk of neurological complications ranges from 4-37\% (Mateen et al. 2011). If VA-ECMO support is used, the patient is endangered by the development of a wide range of both solid and gaseous microemboli. During the pump flow rate increase, blood flows are accelerated with the potential development of turbulent flows. Depending on the pump flow rate parameters, the ascending blood flow from the arterial cannula and the forward flow from the left ventricle can intersect in the area of the aortic arch. 
Table 1.1. Results of the statistical testing of the number of emboli in CCA between 5 ECMO regimes (Friedman) and between all possible pairs of ECMO regimes (Dunn) for all 16 measurements.

\begin{tabular}{|c|c|c|c|c|c|c|c|c|c|c|c|}
\hline \multirow{2}{*}{$\begin{array}{l}\text { Time } \\
(\min )\end{array}$} & \multirow{2}{*}{$\begin{array}{c}\text { Friedman } \\
\mathbf{p}\end{array}$} & \multicolumn{10}{|c|}{ Dunn's test $p$} \\
\hline & & $0 \times 1$ & $0 \times 2$ & $0 \times 3$ & $0 \times 4$ & $1 \times 2$ & $1 \times 3$ & $1 \times 4$ & $2 \times 3$ & $2 \times 4$ & $3 \times 4$ \\
\hline 1 & 0.0001 & - & - & - & $* * *$ & - & - & $* *$ & - & $*$ & - \\
\hline 2 & 0.0001 & - & - & $* *$ & $* * * *$ & - & - & $* *$ & - & $*$ & - \\
\hline 3 & 0.0001 & - & - & $* *$ & $* * * *$ & - & - & $* *$ & - & - & - \\
\hline 4 & 0.0001 & - & - & $* * *$ & $* * * *$ & - & - & $* *$ & - & - & - \\
\hline 5 & 0.0001 & - & - & $* *$ & $* * * *$ & - & - & $* *$ & - & - & - \\
\hline 6 & 0.0001 & - & - & $* *$ & $* * * *$ & - & - & $* *$ & - & - & - \\
\hline 7 & 0.0001 & - & - & $* *$ & $* * * *$ & - & - & $* *$ & - & - & - \\
\hline 8 & 0.0001 & - & - & $* *$ & $* * * *$ & - & - & $* *$ & - & - & - \\
\hline 9 & 0.0001 & - & - & $* *$ & $* * * *$ & - & - & $* *$ & - & - & - \\
\hline 10 & 0.0001 & - & - & $* *$ & $* * * *$ & - & - & $* *$ & - & - & - \\
\hline 11 & 0.0001 & - & - & $* *$ & $* * * *$ & - & - & $* *$ & - & - & - \\
\hline 12 & 0.0001 & - & - & $* * *$ & $* * * *$ & - & - & $* *$ & - & - & - \\
\hline 13 & 0.0001 & - & - & $* * *$ & $* * * *$ & - & - & $* *$ & - & - & - \\
\hline 14 & 0.0001 & - & - & $* * *$ & $* * * *$ & - & - & $* *$ & - & - & - \\
\hline 15 & 0.0001 & - & - & $* *$ & $* * * *$ & - & - & $* *$ & - & - & - \\
\hline 16 & 0.0001 & - & - & $* *$ & $* * * *$ & - & - & $* *$ & - & - & - \\
\hline
\end{tabular}

Table 1.2. Results of the statistical testing of the average blood flow rates in CCA between all 5 ECMO regimes (Friedman) and between all possible pairs of ECMO regimes (Dunn).

\begin{tabular}{cccccccccccc}
\hline $\begin{array}{c}\text { Time } \\
(\mathbf{m i n})\end{array}$ & $\begin{array}{c}\text { Friedman } \\
\mathbf{p}\end{array}$ & $\mathbf{0 x 1}$ & $\mathbf{0 x 2}$ & $\mathbf{0 x 3}$ & $\mathbf{0 x 4}$ & $\mathbf{1 \times 2}$ & $\mathbf{1 x 3}$ & $\mathbf{1 x 4}$ & $\mathbf{2 x 3}$ & $\mathbf{2 \times 4}$ & $\mathbf{3 x 4}$ \\
\hline 1 & 0.0001 & - & $*$ & $* *$ & $* * * *$ & - & - & $* *$ & - & - & - \\
\hline
\end{tabular}

- no significant difference, $*$ significant at significance level of $0.05, * *$ significant at significance level of $0.01, * * *$ significant at significance level of $0.001, * * * *$ significant at significance level of 0.0001 .

These factors together with prothrombogenic stasis of the blood in the left-side heart compartments, and in particular in the aortic root, may, at low cardiac output and along with a serious failure of the left ventricle, result in an increased risk of the development of thrombotic formations with subsequent embolisms into various target organs. Based on the measured results, the flow of blood from the arterial cannula has significant importance with regards to the propagation of embolisms into the CNS, whereby a higher ECMO pump flow rate corresponds to a higher number of embolisms into the CCA, which results in turbulent blood mixing in the aortic arch with the aortic root and arch being washed out and the VA-ECMO arterial cannula becoming the dominant blood supply for the CNS. At high flow rates of the VA-ECMO pump and with simultaneous minimal cardiac output, during which high numbers of embolisms are detected, the blood volume in the CCA is represented in particular by the portion of blood volume from the arterial line. It cannot be excluded that a certain number of microemboli originates from the left heart. However, it may be concluded based on zero VA-CMO flow rate that at least an overwhelming majority of microemboli is corresponded to the application of the VA-ECMO. At high flow rate in the pump, the extracorporeal perfusion system is also unable to perform efficient filtration of the developed microbubbles. From the point of view of the character of the target blood vessels, the magnitude of the flow rate in the CNS and tissue susceptible to hypoxia, the brain is essential. In references there are only a few publications that deal in detail with the issue of microembolism detection using the TCD-proven MES 
during the application of ECMO support. These very rare publications assess the possibility of the propagation of embolisms into the CNS while comparing VV/VA-ECMO (Marinoni et al. 2016) and the risk of MES in patients with left-sided cardiac support. However, these publications include a human study consisting of a very heterogeneous group of patients with various forms of heart failure, various clinical conditions and laboratory parameters and various flow rate regimes under VV/VA-ECMO support. The results of such publications are very difficult to reproduce and the adequate and goal-directed management of the protocol is limited due to concerns for the patients' safety and autonomy. A majority of the publications fail to take into account and assess the importance of a change in the flow rate parameters of the circulation support on the possible development of microembolisms, and only assess the proof of MES related to the application of the given support. The results of publications evaluating the use of ECMO support confirm a higher incidence of microembolisms if VA-ECMO is used compared to VV-ECMO. At high flow rates of the VA-ECMO pump, the higher turbulent flow and flow fluctuations in the aortic arch are basic factors for the occurrence of potential carotid microemboli. Our results confirm the presence of a higher number of emboli with high flow rates under VA-ECMO support (Zanatta et al. 2010). There is one additional publication that deals with an ovine model, the result of which was only mechanistic proof of the propagation of microembolisms into the brain related to the application of ECMO (Zhang et al. 2003). However, there are still few data concerning the incidence of microembolism following ECMO and the aspect of microembolism following ECMO therapy has not been investigated so far. The presented results of our study affirm a higher incidence of microembolisms into the brain during high flow rate regimes under VA-ECMO support. In our study, this concerns the ECMO pump flow rates of 3 and $4 \mathrm{l} / \mathrm{min}$. Other measured data confirm an adequate increase in flow rates in the CCA corresponding to a change in the VA-ECMO flow rates. Management of the VA-ECMO pump flow rate is of primary importance in terms of influencing the hemodynamic parameters in the given patient. Regulation of the pump flow rate is critical not only for perfusion pressure in the organism, but also for the potential risk of development of embolisms and their distribution into the CNS during various flow rate parameters under VA-ECMO. Differing the parameters of the pump flow rate may aggravate the neurological status of predisposed patients depending on the number and character of embolisms. High ECMO flow rate regimes may not have a primary beneficial effect on the perfusion pressure and circulation parameters. They may also have a negative impact on the resulting neurological status of the patient. The highest ECMO flow rates in cardiogenic shock negatively affect also left ventricle as was clearly shown by several authors and it seems that ECMO flow should be set as low as possible to maintain adequate tissue perfusion.

\section{Conclusions}

Based on the evaluated results, it is clear that high VA-ECMO pump flow rates are related to a statistically significant risk of embolisms into the CNS compared to minimal pump flow rates. If high flow rates are used for the VA-ECMO support, the risk of embolism is higher compared to the standard measurements of embolism without the VA-ECMO support.

\section{Conflict of Interest}

There is no conflict of interest.

\section{Acknowledgements}

This work was supported by grant projects No. SVV 260379 and Progres Q38.

\section{References}

AASLID R, MARKWALDER TM, NORMES H: Noninvasive transcranial Doppler ultrasound recording of flow velocity in basal cerebral arteries. J Neurosurg 57: 769-774, 1982.

AUSTEN WG, HOWRY DH: Ultrasound as method to detect bubbles or particulate matter in the arterial line during cardiopulmonary by-pass. J Surg Res 5: 283-284, 1965.

BARTLETT RH, GATTINONI L: Current status of extracorporeal life support (ECMO) for cardiopulmonary failure. Minerva Anestesiol 76: 534-540, 2010. 
CHANG CH, CHEN HC, CAFFREY JL, HSU J, LIN JW, LAI MS, CHEN YS: Survival analysis after extracorporeal membrane oxygenation in critically ill adults a nationwide cohort study. Circulation 133: 2423-2433, 2016.

DROSTE DW, MARKUS HS, NASSIRI D, BROWN MM: The effect of velocity on appearance of embolic signal studied in transcranial Doppler models. Stroke 25: 986-991, 1994.

HÁLA P, MLČEK M, OŠŤÁDAL P, JANÁK D, POPKOVÁ M, BOUČEK T, LACKO S, KUDLIČKA J, NEUŽIL P, KITTNAR O: Regional tissue oximetry reflects changes in arterial flow in porcine chronic heart failure treated with venoarterial extracorporeal membrane oxygenation. Physiol Res 65 (Suppl 5): S621-S631, 2016.

KUDO T, INOUE Y, SUGANO N, IWAI T: Doppler ultrasound for detection of microembolic signals in peripheral arteries. Eur J Vasc Endovasc Surg 24: 37-42, 2002.

MADERSHAHIAN N, NAGIB R, WIPPERMANN J, STRAUCH J, WAHLERS T: A simple technique of distal limb perfusion during prolonged femoro-femoral cannulation. J Card Surg 21: 168-169, 2006.

MAKDISI G, WANG IW: Extra Corporeal Membrane Oxygenation (ECMO) review of a lifesaving technology. J Thorac Dis 7: E166-E176, 2015.

MARINONI M, MIGLIACCIO ML, TRAPANI S, BONIZZOLI M, GUCCI L, CIANCHI G, GALLERINI A, TADINI BUONINSEGNI L, CRAMARO A, VALENTE S, ET AL.: Cerebral microemboli detected by transcranial doppler in patients treated with extracorporeal membrane oxygenation. Acta Anaesthesiol Scand 7: 934-944, 2016.

MARKUS HS, MACKINNON A: Asymptomatic embolisation detected by Doppler ultrasound predicts stroke risk in symptomatic carotid artery stenosis. Stroke 36: 971-975, 2005.

MATEEN FJ, MURALIDHARAN R, SHINOHARA RT, PARISI JE, SCHEARS GJ, WIJDICKS EF: Neurological injury in adults treated with extracorporeal membrane oxygenation. Arch Neurol 68: 1543-1549, 2011.

NADAREISHVILI ZG, CHOUDARY Z, JOYENER C, BRODIE D, NORRIS JW: Microemboli detection in acute myocardial infarction. Stroke 30: 2679-2682, 1999.

OSTADAL P, MLCEK M, STRUNINA S, HRACHOVINA M, KRUGER A, VONDRAKOVA D, JANOTKA M, HALA P, KITTNAR O, NEUZIL P: Novel porcine model of acute severe cardiogenic shock developed by upper-body hypoxia. Physiol Res 65: 711-715, 2016.

RINGELSTEIN EB, DROSTE DW, BABIKIAN VL, EVANS DH, GROSSET DG, KAPS M, MARKUS HS, RUSSELL D, SIEBLER M: Consensus on microembolus detection by TCD. International Consensus Group on Microembolus Detection. Stroke 29: 725-729, 1998.

ZANATTA P, FORTI A, BOSCO E, SALVADOR L, BORSATO M, BALDANZI F, LONGO C, SORBARA C, IONGATTI P, VAFRÉ C: Microembolic signals and strategy to prevent gas embolism during extracorporeal membrane oxygenation. Cardiothorac Surg 5: 5, 2010.

ZHANG J, FAN XM, SONG GW, YIN HH, GAN XZ, LI H, QIAN SY: Transcranial Doppler monitoring of cerebral blood flow velocity during extracorporeal membrane oxygenation in sheep. (In Chinese) Zhonghua Yi Xue Za Zhi 83: 872-876, 2003. 